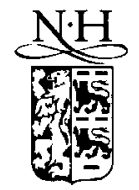

ELSEVIER

\title{
Energy straggling and multiple scattering in silicon strip detectors
}

\author{
T. Antičicic ${ }^{b}$, R. Battiston ${ }^{d}$, W. Braunschweig ${ }^{a}$, Y.H. Chang ${ }^{c}$, C.-Y. Chien ${ }^{b}$, A.E. Chen ${ }^{c}$, \\ S.R. Hou ${ }^{\mathrm{c}, *}$, C.H. Lin ${ }^{\mathrm{c}}$, W.T. Lin ${ }^{\mathrm{c}}$, R. Ostonen ${ }^{\mathrm{e}}$, K. Spartiotis ${ }^{\mathrm{e}}$, O. Syben ${ }^{\mathrm{a}}$, O. Toker ${ }^{\mathrm{d}, 1}$, \\ B. Wittmer ${ }^{\mathrm{a}}$ \\ a I. Physikalisches Institur, RWTH, D-52056 Aachen, Germany \\ ${ }^{\mathrm{h}}$ Department of Physics and Astronomy, Johns Hopkins University, Baltimore, MD 27218, USA ${ }^{2}$ \\ ${ }^{c}$ Physics Department, National Central University, Chungli, 32054 Taiwan ${ }^{3}$ \\ ${ }^{d}$ INFN-Sezione di Perugia and Universitá Degli Studi di Perugia, 1-06100 Perugia, Italy \\ ' SEFT, Research Institute for High Energy Physics, P.O. Box 9, SF-00014 Helsinki. Finland
}

Received 23 January 1996

\begin{abstract}
We present a test beam study of energy straggling and multiple scattering in silicon strip detectors using electrons and pions of momenta up to $50 \mathrm{GeV}$. Results are compared with GEANT simulation using a simple algorithm to parameterize energy loss distribution. The deflection due to multiple scattering in crystalline structure was investigated by placing a GaAs wafer at various angles.
\end{abstract}

\section{Introduction}

Silicon strip detectors are widely used in tracking devices because of their high spatial resolution and stable operation. The tracking precision of silicon detector depends on ionization energy loss measurement and deflection by multiple scattering, therefore good understanding of these phenomena is essential. In this report we present a test beam analysis of energy straggling and multiple scattering in silicon strip detectors.

Energy loss straggling in thin absorbers was first studied by Landau and Vavilov [1]. We have applied full GEANT [2] simulation to calculate the energy loss in a silicon strip detector using Urbán [3] and photoabsorption ionization (PAI) [4] models. The measured energy loss is fitted to a Gaussian convoluted Landau distribution and characterized by three fitting parameters. Comparisons between data and simulation are made for all beam line configurations.

Multiple Coulomb scattering is the counterpart to high spatial resolution of silicon strip detectors. The residuals

\footnotetext{
* Corresponding author. Present address: CERN, CH-1211 Geneva 23, Switzerland. Tel. +41227679358 , fax +41227828923 , e-mail suenhou@vxcern.cern.ch.

${ }^{\prime}$ Now at IDE AS, Gaustadalléen 21, N-0371 Oslo, Norway.

${ }^{2}$ Work supported by National Science Foundation (PHY-9221761).

${ }^{3}$ Work supported by National Science Council (NSC83-0208-M-008-047) and Tsu Yuan-Chi memorial foundation.
}

of measured impact position from the projection of linear track fitting are compared with GEANT calculations using Molière [5] theory. The deflection of beam particles due to the crystalline structure was studied by placing a GaAs wafer at various angles in the beam line.

\section{Test beam setup}

The test beam study was conducted at $\mathrm{X} 3$ complex of CERN-SPS, using electrons of $4,10,25$ and $50 \mathrm{GeV}$ and pions of $50 \mathrm{GeV}$. Events were triggered by coincidence of scintillation counters with a beam spot dimension of $1 \times 1 \mathrm{~cm}^{2}$. The beam momentum spread is dominated by the opening width of collimators, wider opening was applied at low beam momentum to gain high event rate with $\delta p / p=4 \%$ and $1 \%$ for beam particle momenta of 4 and $50 \mathrm{GeV}$ respectively. The electron beam has high purity (above $97 \%$ ). The beam contamination is monitored by a downstream calorimeter for electron and pion (MIP) identification.

Parameters of the six silicon strip detectors used in this study are listed in Table 1 . The $Y_{\mathrm{S}}$ and $Y_{\mathrm{N}}$ are ladders of long strip length. $X_{1.2}$ and $Y_{1.2}$ with strip length of $20 \mathrm{~mm}$ have low electronic noise that matches well with the Viking circuits [6] employed for readout. High signal to noise ratio is obtained. The data acquisition is an IBM-PC based system interfaced to SRS-SDA modules [7] with 8 bit ADC dynamic range. The beam line setup is illustrated in Fig. 1. 
Table 1

The geometrical parameters of detectors

\begin{tabular}{|c|c|c|c|c|c|c|c|}
\hline & $X_{!}$ & $Y_{1}$ & $X_{2}$ & $r_{2}$ & GaAs & $Y_{\mathrm{S}}$ & $Y_{\mathrm{N}}$ \\
\hline$\therefore-$ position $|\mathrm{mm}|$ & 76.5 & 84.4 & 108.3 & 116.2 & 73.5 .0 & 1035.0 & 11350 \\
\hline Thickness $|\mu \mathrm{m}|$ & 300 & 300 & 300 & 300 & 500 & 300 & 320 \\
\hline Active width $|\mathrm{mm}|$ & 19.2 & 19.2 & 19.2 & 19.2 & - & 6.4 & 6.4 \\
\hline Strip length $[\mathrm{mm}]$ & 20 & 20 & 20 & 20 & - & $2 \times 60$ & $4 \times 80$ \\
\hline Strip pitch $\mid \mu \mathrm{m}]$ & 25 & 25 & 25 & 25 & - & 50 & 25 \\
\hline Readout pitch $[\mu \mathrm{m}]$ & 50 & 50 & 50 & 50 & - & 50 & 50 \\
\hline Manufacturer & CSEM & CSEM & CSEM & CSEM & Freiberger & VTT & ERSO \\
\hline
\end{tabular}

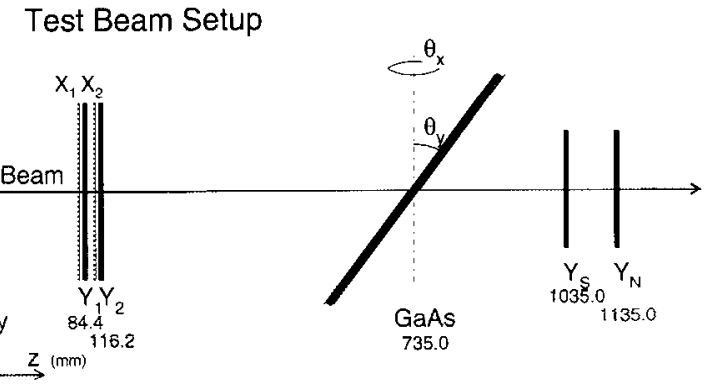

Fig. 1. Beam line setup.

\section{Data processing}

We have sampled pedestal events between beam spills during data taking. For each beam line configuration relative pedestal values of all readout channels were analyzed with noise level $\left(\sigma_{\mathrm{N}}\right)$ of each detector determined as the average RMS of all pedestals. The common shift was uniform over one Viking chip. The charge collected by each readout strip is the net ADC after pedestal and common shift subtraction.

The ionization charge generated by the traversing beam particle in silicon wafer drifts to one or more adjacent strips to form a cluster. In this analysis a cluster is defined with the charge of peak strip above $3 \sigma_{\mathrm{N}}$ and the neighboring strips with charge above $1 \sigma_{\mathrm{N}}$. The total sum is required to be larger than $6 \sigma_{\mathrm{N}}$. The signal-to-noise ratios obtained are listed in Table 2, along with the corresponding noise levels.

For clusters containing more than one strip, the cluster chargc is divided into the sum of left and right strips $\left(Q_{r}, Q_{1}\right)$ divided by the cluster center calculated by center-of-gravity. The event distribution $f(\eta)$, with $\eta=Q_{\mathrm{r}} /\left(Q_{\mathrm{r}}+Q_{1}\right)$, characterizes the nonlinear charge sharing between strips [8]. Assuming the incident beam particles are uniformly distributed between readout strips, the impact position is obtained according to the $\eta$ value by

$$
y=y_{0}+p \int_{1}^{\eta} f(\eta) / \int_{0}^{1} f(\eta)
$$

where $p$ is the readout pitch and $y_{n}$ the strip offset. The geometrical calibration for all delectors included the vertical offset and the tilt in the transverse plane.
The dominant systematic uncertainty of spatial resolution is the multiple scattering. The residuals of linear track fitting are contributed by multiple scattering $\left(\sigma_{\mathrm{ms}}\right)$ and intrinsic resolution of the detector $\left(\sigma_{\mathrm{int}}\right)$. We have performed GEANT simulation for multiple scattering with an additional Gaussian smearing of the hit positions to account for intrinsic resolution. The intrinsic resolutions were determined by varying the smearing widths until agreements were found between the simulation and data on the residuals of linear track fitting [9]. Calibration data of $50 \mathrm{GeV}$ electrons were used and the intrinsic resolutions obtained are also listed in Table 2.

\section{Energy straggling}

The total cluster charge collected by a silicon strip detector corresponds to the ionization energy loss of the traversing charged particle. The energy loss fluctuations in a silicon wafer of typical thickness of $300 \mu \mathrm{m}$ are described by the Gaussian convoluted Landau distribution [10] given by

$$
\begin{aligned}
& f(\Delta, x)= \\
& \frac{1}{\sqrt{2 \pi} \sigma} \int_{-\infty}^{+\infty} \exp \left(-\frac{\left(\Delta-\Delta^{\prime}\right)^{2}}{2 \sigma^{2}}\right) f_{\mathrm{L}}\left(\Delta^{\prime}, x\right) \mathrm{d} \Delta^{\prime} .
\end{aligned}
$$

The Landau density function is

$$
\begin{aligned}
f_{\mathrm{L}}(\Delta, x) & =\frac{1}{\xi} \phi(\lambda), \\
\phi(\lambda) & =\frac{1}{2 \pi \mathrm{i}} \int_{\sigma-i \infty}^{\sigma+i \infty} \exp (\lambda u+u \ln u) \mathrm{d} u,
\end{aligned}
$$

where $\lambda=\left(\Delta-\Delta_{\mathrm{mp}}\right) / \xi$. In practice we use

$$
f(\Delta, x)=\mathcal{C} \sum_{\Delta-4 \sigma}^{\Delta+4 \sigma} \exp \left(-\frac{\left(\Delta \cdots \Delta^{\prime}\right)^{2}}{2 \sigma^{2}}\right) \phi(\lambda) \delta \Delta^{\prime},
$$

with DENLAN [11] used for $\phi(\lambda)$. Relevant fitting parameters are $\Delta_{\mathrm{mp}}$, the most probable value; $1 / \xi$ that characterizes the distribution width; and $\sigma$ of the convoluting Gaussian function. 
Table 2

Performance of silicon strip detectors.

\begin{tabular}{|c|c|c|c|c|c|c|}
\hline & $X_{1}$ & $Y_{1}$ & $X_{2}$ & $Y_{2}$ & $Y_{S}$ & $y_{\mathrm{N}}$ \\
\hline Signal-to-noise & 51.8 & 56.2 & 66.5 & 53.3 & 26.7 & 13.8 \\
\hline Noise level [ADC] & 1.3 & 1.3 & 1.0 & 1.3 & 1.4 & 4.3 \\
\hline Intrinsic resolution $\left.\sigma_{\mathrm{int}} \mid \mu \mathrm{m}\right]$ & - & 3.2 & - & 3.2 & 6.0 & 5.0 \\
\hline
\end{tabular}

The $\eta$ variable is the dominant parameter to study the position dependence of energy straggling spectrum between readout strips. In Fig. 2a, the $\eta$ distribution of $Y_{1}$ is shown for all the test beam configurations; $\eta=0$ and 1 correspond to positions of neighboring readout strips and the bump at $\eta=$ 0.5 corresponds to the presence of floating strip. Events in $|\eta-0.5|>0.3$ have large portion of cluster charge taken by the nearest readout strip that face the limited ADC dynamic rangc with lowcr average cluster charge obtained (Fig. 2b). This is particularly true for clusters containing two strips total (dark shaded histogram). The cluster charge spectra in three $\eta$ fractions of $|\eta-0.5|<0.3$ that have no ADC overflow problem are shown in Fig. 3. The corresponding parameters of the Gaussian convoluted Landau fit of them are illustrated in Fig. 4 in which the horizontal lines are the values obtained on spectra containing all three $\eta$ fractions.

The two parameters used to characterize the energy loss fluctuation are i) the ratio of typical energy loss to the maximum transferable energy in a single collision $(\kappa=$ $\xi\left(\epsilon_{\mathrm{m}}\right)$; and ii) the number of low-energy collisions $(\delta=$ $\min (t(\mathrm{~d} E / \mathrm{d} x) / I, \xi / I))$. For a silicon wafer thickness of

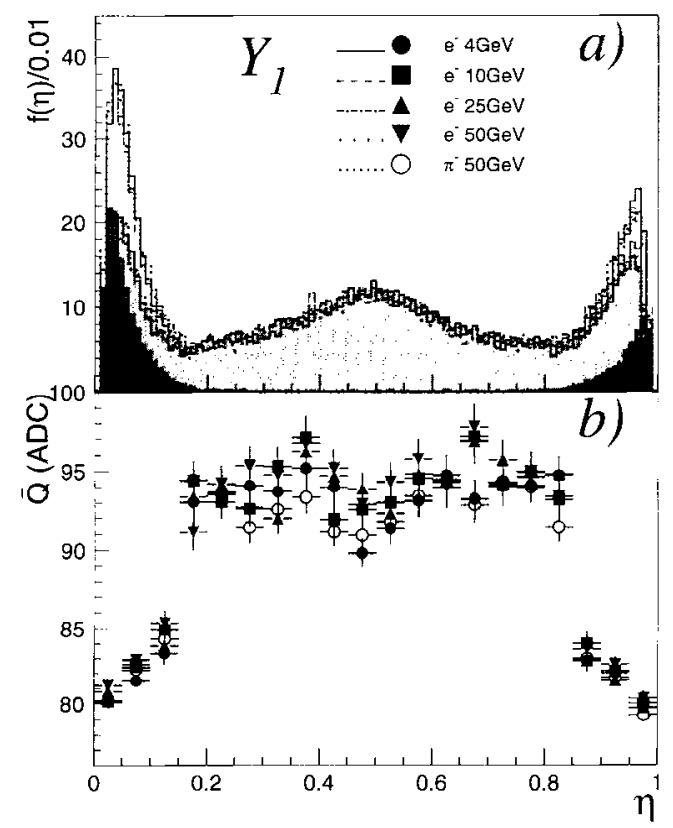

Fig. 2. (a) $\eta$ spectrum of $y_{1}$ clusters; classified into constituents of clusters of two-strips (dark shaded) and more than two strips (light shaded). (b) the average cluster charge versus $\eta$.
$300 \mu \mathrm{m}$, we have $\delta \simeq 30$ for both electrons and pions of momenta above $1 \mathrm{GeV}$. The GEANT calculation would consider the ionization process as via small number of low energy collisions with Urbán and PAI models provided for simulation. We have performed the GEANT simulation with no explicit $\delta$-ray generation and the electron and photon cut off thresholds were set to $100 \mathrm{keV}$. The tracking steps are calculated automatically (AUTO $=\mathrm{I}$ ) according to the tracking parameters chosen. The results are found to be independent of the numbers of steps taken in silicon wafer. The relevant tracking and steering parameters are listed in Table 3.

By a simple scaling mechanism, the energy loss simulated as the sum of $\mathrm{dE} / \mathrm{d} x$ deposits is converted to the ADC reading by

$Q=\Delta_{\mathrm{mp}}+W\left(S \sum \frac{\mathrm{d} E}{\mathrm{~d} x}-\Delta_{\mathrm{mp}}\right)$

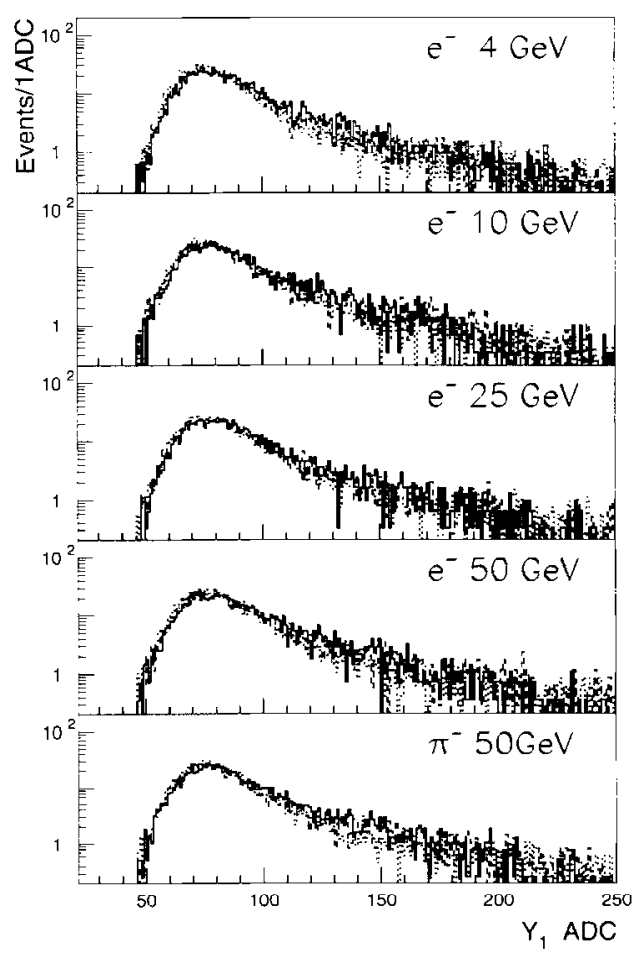

Fig. 3. Distributions of cluster charge of different $\eta$ fraction: events of $|\eta-0.5|$ in $(0.0 .0 .1)$ are presented by dotted-line, $(0.3 .0 .2)$ by dash-line. and $(0.2,0.3)$ by solid-line. 


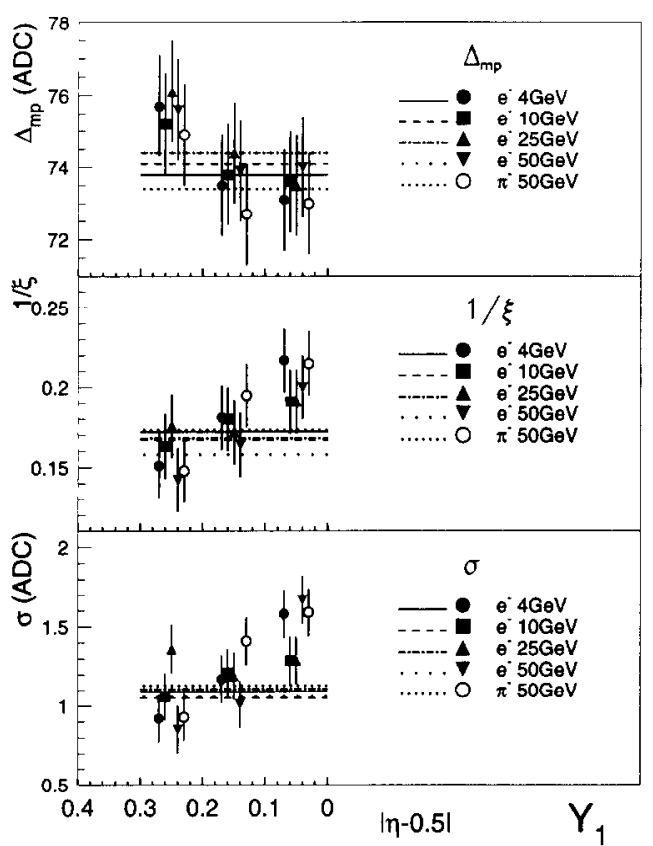

Fig. 4. Parameters of Gaussian convoluted Landau fit to the cluster charge of different fraction of $\eta$

Table 3

GEANT tracking and steering parameters

STEMAX STMIN EPSIL CUTGAM CUTELE AUTO LOSS MULS

$10^{10} \mathrm{~cm} \quad 0 \mathrm{~cm} \quad 10 \mu \mathrm{m} \quad 100 \mathrm{keV} \quad 100 \mathrm{keV} \quad 1 \quad 2 \quad 2$

where $\Delta_{m p}$ is the most probable value from Gaussian convoluted Landau fit, the scaling parameter $S$ converts $\sum(\mathrm{d} E / \mathrm{d} x)$ to $\mathrm{ADC}$ counts and $W$ adjusts the width to agree with data. Calibration on $S$ and $W$ was performed on $Y_{\text {I }}$ sensor using data of $50 \mathrm{GeV}$ electron beam and applied to all detectors. The values applied for both Urbán and PAI models are listed in Table 4 . In addition, the noise level is added as a Gaussian smearing of $4 \mathrm{ADC}$ counts for $Y_{\mathrm{N}}$ and 1 ADC count for the rest.

The energy loss distributions of $Y_{1}$ in $|\eta-0.5|<0.3$ of all beam line configurations are shown in Fig. 5 . The corresponding parameters of Gaussian convoluted Landau fit obtained are illustrated in Fig. 6. Since the parameters applied were calibrated by $50 \mathrm{GeV}$ electrons, discrepancies increase at low beam momentum. The PAI model gives a more detailed calculation on energy loss straggling with better agreement in the high charge tails. This is also seen in the fitting of the $1 / \xi$ and $\sigma$ parameters.

\section{Multiple scattering}

To study the effect of multiple scattering to the spatial resolution, we compare the residuals of detectors measuring

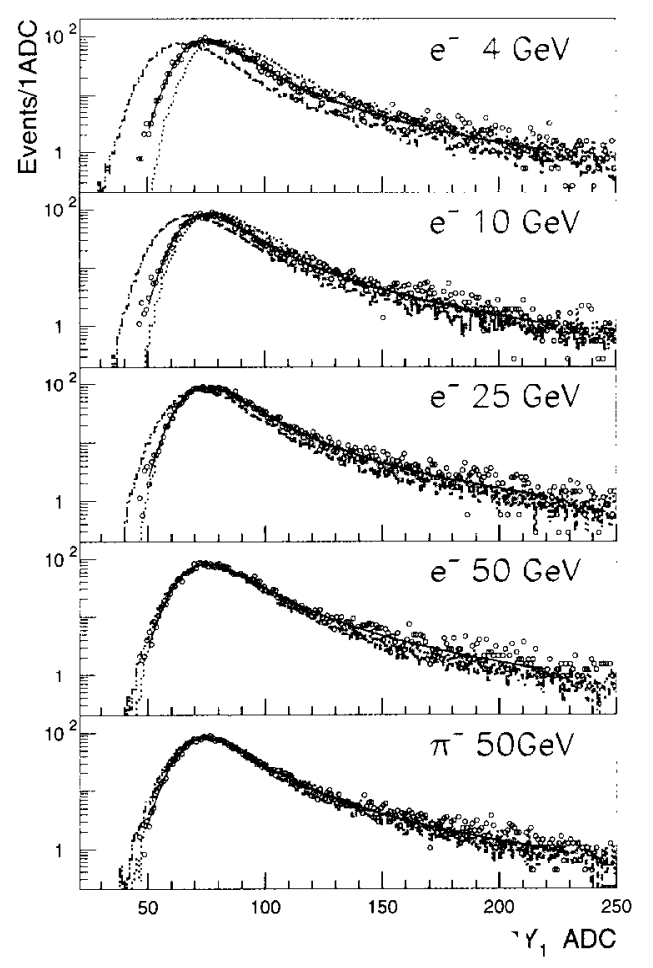

Fig. 5. Distributions of cluster charge (circles); histograms are simulation of Urbán (dash-dotted lines) and PAI (dotted lines) models; solid lines are the fit to Gaussian convoluted Landau distribution.

$y$ coordinate to the unweighted linear track fitting. A passive $\mathrm{GaAs}$ wafer is positioned in the middle of the test beam setup with rotation angles to the $x$ and $y$ axis at $\left(\theta_{x}, \theta_{y}\right)$. The rotation has $\theta_{x}$ conducted on $x-z$ plane followed by $\theta_{y}$

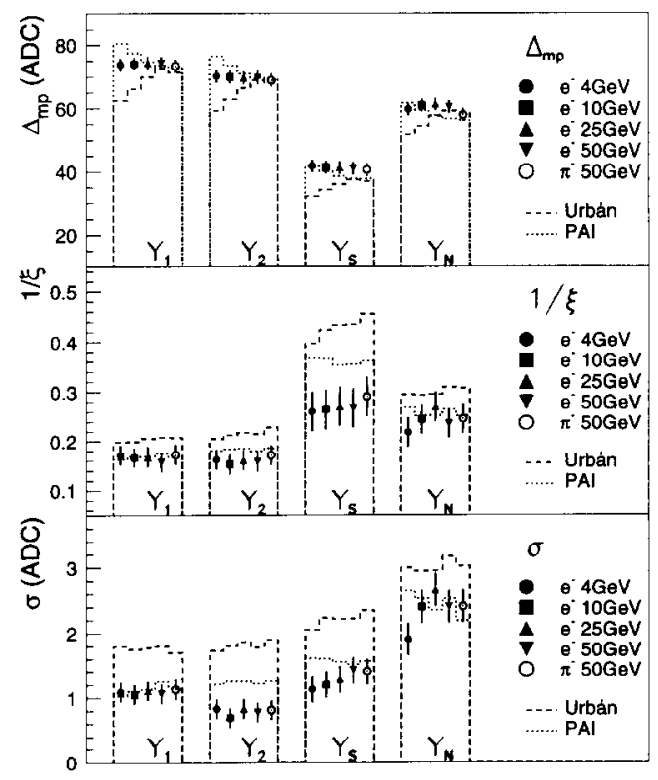

Fig. 6. Parameters of Gaussian convoluted Landau fit to the cluster charge spectra; histograms are results of fit to GEANT simulation. 
Table 4

Parameters that convert jonization energy loss simulation to ADC reading

\begin{tabular}{llrr}
\hline & STRA & \multicolumn{1}{c}{$S$} & $W$ \\
\hline Uibán & 0 & 9180 & 2.2 \\
PAI & 1 & 12100 & 1.2 \\
\hline
\end{tabular}

around the new $x$-axis. At $\theta_{x}=0$ and $\theta_{y}=0$ the wafer plane is perpendicular to beam line. The GaAs wafer is $500 \mu \mathrm{m}$ in thickness $\left(0.022 X_{0}\right)$, and the wafer surface is a (100) crystallographic plane.

The GEANT simulation performed has tracking steps calculated automatically according to the tracking parameters of Table 3, and the intrinsic resolutions (Table 2) are applied by Gaussian smearing for each detector. The variable corresponding to the number of collisions is $\Omega_{0}=b_{c} z^{2} t / \beta^{2}$ where $z$ is the charge of the incident particle traversing at speed $\beta, t$ is the absorber thickness and $b_{c}$ is a constant For electrons and pions of momentum above $4 \mathrm{GeV}$ traversing $300 \mu \mathrm{m}$ of silicon $\left(\Omega_{0}\right.$ is an order of magnitude larger than the threshold of $\Omega_{0}>20$ ), the Molière theory is chosen $(M U L S=2$ ) to calculate the polar angle deflection. The results obtained are independent of the number of steps taken in the silicon wafer. The planar (one-dimensional) deflection angle of Molière's theory is roughly Gaussian with high tails. The RMS of the angular distribution is formulated by $[12]$

$\theta_{0}=\frac{13.6 \mathrm{MeV}}{\beta c p} z \sqrt{\frac{t}{X_{0}}}\left[1+0.038 \ln \left(\frac{t}{X_{0}}\right)\right]$.

At $50 \mathrm{GeV}$, the multiple scattering has magnitude comparable to the detector intrinsic resolution; it dominates at lower beam energy.

We have performed unweighted linear track fitting on both data and GEANT simulation. Along the beam line, the $Y_{1}$ and $Y_{2}$ are positioned close to each other upstream to the GaAs wafer and the $Y_{\mathrm{S}}$ and $Y_{\mathrm{N}}$ are downstream with larger spacing. Such an arrangement provides stiff fitting to the upstream measurements and equal amplification to the $Y_{S}$ and $Y_{\mathrm{N}}$ measurements. The RMS of the residuals obtained are illustrated in Fig. 7 for all beam line setups. Good agreement is seen between data and GEANT simulation for beam line setups with $\mathrm{GaAs}$ wafer at $\theta_{y}=0$ when the GaAs wafer is parallel to the $y$-axis of the detector measuring coordinate. The multiple scattering calculated by GEANT assumed the tracking media is homogeneous. We have observed larger residuals for GaAs wafer at $\theta_{y}=45^{\circ}$ to the GEANT simulation. This is due to the crystalline structure not simulated by GEANT.

\section{Summary}

The high signal-to-noise performance of silicon strip detectors in this study provided a good comparison of energy

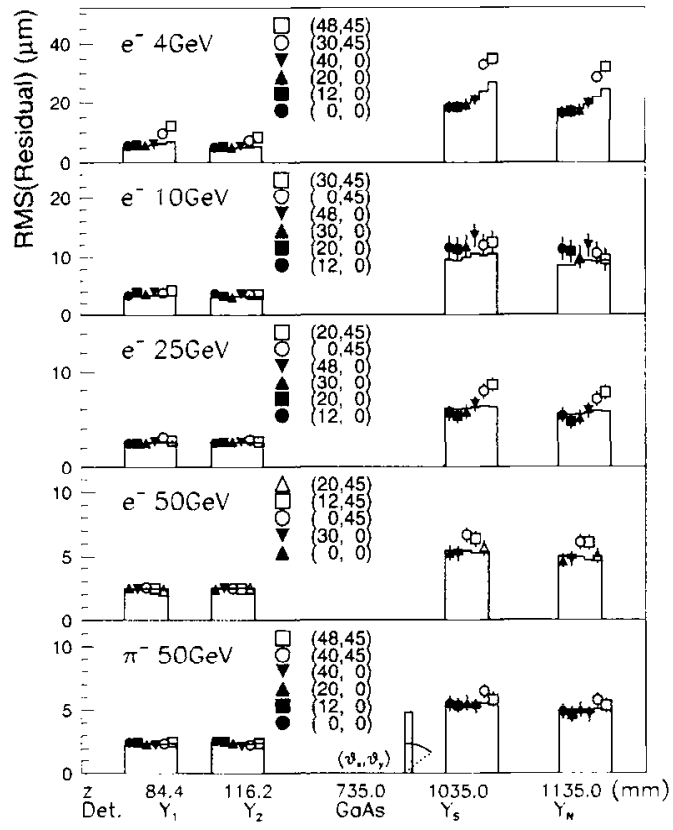

Fig. 7. Residuals of measured $y$ to the linear track fitting. The GaAs angles are labeled by $\left(\theta_{x}, \theta_{y}\right)$ to the $x$ and $y$-axis respectively; histograms are GEANT simulation.

loss straggling to the models of GEANT with a simple scaling mechanism to convert simulation on $\mathrm{d} E / \mathrm{d} x$ strip cluster charge. The more detailed PAI model gives better agreement with data than the Urbán model.

The systematic uncertainty caused by multiple scattering is the major concern for application in tracking devices. The passive GaAs wafer positioned in the beam line provided crystalline material to magnify the effect of multiple scattering. Comparison with the GEANT simulation of beam momenta between 4 to $50 \mathrm{GeV}$ has shown good agreement when the GaAs wafer plane is parallel $\left(\theta_{y}=0\right)$ to the axis of the detector measuring coordinate. Larger residuals are seen when the GaAs plane is set at $\theta_{y}=45^{\circ}$. This indicates the effect of crystalline structure not included in GEANT.

\section{References}

[1] L. Landau. J. Phys. (Moscow) 8 (1944) 201: P.V. Vavilov, Zh. Eksp. Teor. Fiz. 32920 ( 1957 ); Sov. Phys. JETP 5 (1957) 749 .

[2] GEANT Version 3.21 (March 1994); R. Brun et al.. CERN DD/EE/84-1 (September 1987). CERN Program Library Long Writeup W5013 (March 1994).

[3] L. Urbán, GEANT 3.16 PHYS332 (revised December 1993], CERN Program Library Long Writeup W5013 (March 1994).

[4] I. Gavrilenko et al., GEANT 3.21 PHYS334 (revised March 1994). CERN Program Library Long Writeup W5013 (March 1994).

15] Z. Molière, Z. Naturforsch. 2a (1947) 133; H.A. Bethe, Phys. Rev. 89 (1953) 1256; W.T. Scott, Rev. Mod. Phys. 35 (1963) 231 : and Shen et al., Phys. Rev. D 20 (1979) 1584.

[6] O. Toker et al., Nucl. Instr, and Meth. A 340 (1994) 572. 
17| F.A. Kirsten and C. Haber, IEEE Trans. Nucl. Sci. NS-37 (1990) 288.

|8| E. Belau et al., Nucl. Instr. and Meth. 214 (1983) 253.

191 Y.H. Chang et al., Nucl. Instr. and Meth. A 363 (1995) 5.38.

101 O. Blunck and S. Leisegan. Z. Phys. 128 (1950) 500;

U. Fano. Annu, Rev. Nucl. Sci. 13 ( 1963) 201:

H. Bichsel and P. Saxon. Phys. Rev. A 11 (1975) 1286; and
S. Hancock et al., Phys. Rev. A 28 (1983) 615.

[11] K.S. Koelbig. CERNLIB G110 (August 1985): and

K.S. Koelbig and B. Schorr. Comput. Phys. Commun. 31 (1984) 97

112 V.L. Highland, Nucl. Instr. and Meth. 129 (1975) 497; Nucl, Instr. anc Meth. 161 (1979) 171

G.G. Lynch and O.I. Dahl, Nucl. Instr. and Meth. B 58 (1991) 6. 